\title{
Infección del sitio quirúrgico en niños sometidos a cirugía cardíaca con cierre esternal diferido. Estudio de casos y controles
}

\author{
Javiera Retamal, Pedro Becker, Rodrigo González, Marcela Ferrés, Jaime Cerda, \\ María I. Riquelme, Regina Pérez y Cristián Clavería
}

\section{Surgical site infections in children undergoing cardiac surgery with delayed sternal closure. Case-control study}

Introduction: Surgical site infections (SSI) are an important cause of morbidity in pediatric cardiac surgery. Risk factors in patients requiring delayed sternal closure (DSC) are unknown. Aim: To report the rate of SSI in children undergoing cardiac surgery with DSC and determine the risk factors. Methodology: A retrospective case-control study, in patients younger than 15 years old undergoing cardiac surgery with DSC in our center between 2009 and 2010. SSI was diagnosed according to the criteria of the nosocomial infections committee of our institution, based on international recommendations. Univariate and multivariate analysis of variables was performed. A p $<0.05$ was considered significant. Results: 58 patients were included; the average age was 9.5 days. The most frequent diagnosis were transposition of the great arteries (36\%) and hypoplastic left heart syndrome (27\%). 13 patients had SSI (22\%); 11 incisional and 2 mediastinitis. It was independently associated to SSI by-pass (BP) time longer than 200 min (OR adjusted =9,53; IC 95\% 1,37-66,35) and mechanical ventilation (MV) more than 5 days (OR adjusted $=8,98$; IC 95\% 1,16-69,40). Conclusion: The duration of BP and MV are risk factors of SSI in children undergoing cardiac surgery with DSC.

Key words: Surgical site infection, delayed sternal closure, cardiac surgery, congenital heart disease.

Palabras clave: Infección del sitio quirúrgico, cierre esternal diferido, cirugía cardíaca, cardiopatías congénitas.

\section{Introducción}

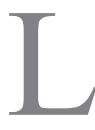

as infecciones del sitio quirúrgico (ISQ) son las infecciones nosocomiales más frecuentes en pacientes post operados ${ }^{1,2}$. Aumentan los días de hospitalización, los costos asociados a la intervención, prolongan el uso de antimicrobianos, aumentan significativamente el riesgo de mortalidad y son consideradas un marcador de calidad de la atención ${ }^{1,3-6}$.

Las ISQ son una importante causa de morbilidad en pacientes pediátricos sometidos a cirugía cardíaca. La tasa reportada es variable y oscila entre 3 y $8 \%{ }^{6-8}$. En análisis retrospectivos se han identificado como factores de riesgo asociados a ISQ la duración de la cirugía, la edad menor de 1 mes, la duración del requerimiento de inótropos y la ventilación mecánica invasora (VMI), entre otros ${ }^{9-14}$.

El cierre esternal diferido (CED) es una técnica ampliamente utilizada en cirugía cardíaca de alta complejidad. Consiste en el cierre de la esternotomía media en un tiempo quirúrgico posterior al de la cirugía cardíaca realizada, e implica el manejo del paciente en los primeros días del postoperatorio en la Unidad de Cuidados Intensivos Pediátrica (UCIP) con la esternotomía abierta y cubierta por una membrana de polietileno suturada a la piel (Figuras 1a y 1b). Es una alternativa eficaz para evitar el efecto constrictivo torácico durante el post-operatorio de cirugías cardíacas complejas asociadas a edema pulmonar y cardíaco y presencia de dispositivos extra-cardíacos que ocupan espacio (por ejemplo, conductos entre el ventrículo derecho y arterias pulmonares) ${ }^{15}$, y facilita el manejo de pacientes con sangrado durante el post operatorio inmediato. Publicaciones recientes respaldan su indicación de rutina en intervenciones paliativas como la cirugía de Norwood ${ }^{16}$; sin embargo, su uso se ha asociado a mayor frecuencia de infecciones en el post operatorio, entre ellas, ISQ y especialmente bacteriemias ${ }^{17,18}$ y a hospitalizaciones más prolongadas. Además, la evidencia ha fallado en demostrar un beneficio en la disminución de la mortalidad post quirúrgica de estos pacientes ${ }^{19}$. Pero sí se han reportado beneficios desde el punto de vista hemodinámico y ventilatorio ${ }^{7,19,20}$.

La tasa de ISQ reportada en los pacientes sometidos a CED es muy variable ( 1 a $28 \%$ ) atribuidas principalmente a las condiciones de gravedad en su post operatorio inmediato, tipo de profilaxis antimicrobiana recibida y determinados diagnósticos de la cardiopatía de base que
Pontificia Universidad Católica de Chile, Santiago. Facultad de Medicina División de Pediatría (JR, MF, RP, CC) División de Enfermedades Cardiovasculares (PB, RG). Departamento de Salud Pública (JC) Hospital Clínico, Red de Salud UC CHRISTUS.

Enfermera Comité de Prevención y Control IAAS (MR).

Declaración de conflictos de interés: Ninguno

Fuente de financiamiento: Ninguno

Recibido: 11 de abril de 2016

Aceptado: 5 de julio de 2016

Correspondencia a: Cristián Clavería Rodríguez claveria@med.puc.cl 


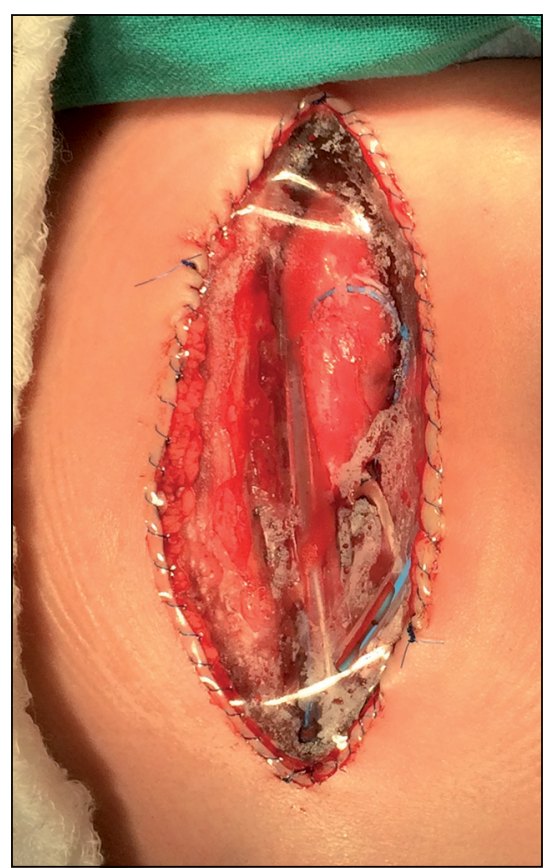

Figura 1a. Recién nacido post operado de cirugía cardíaca con esternotomía abierta, la que está cubierta por una membrana de polietileno estéril suturada a los bordes de la piel.

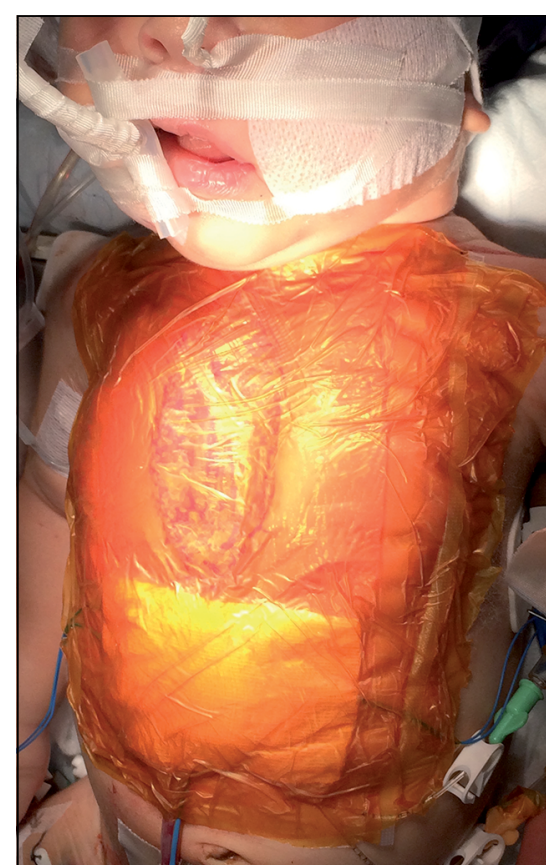

Figura $1 b$. Recién nacido post operado de cirugía cardíaca con esternotomía abierta, cubierta por una membrana de polietileno estéril suturada a los bordes de la piel y sellada, por sobre la membrana, con varias capas de lovan ${ }^{\circledR}$.

se asocian a un mayor riesgo (doble salida del ventrículo derecho, troncos arteriosos, transposición de grandes arterias, principalmente) $)^{6,21-23}$.

Existe aún escasa información en la literatura científica respecto a los factores de riesgo de ISQ en pacientes con CED.

El objetivo de este estudio fue evaluar la tasa de ISQ en los pacientes portadores de cardiopatía congénita sometidos a cirugía cardíaca con CED y determinar si los factores de riesgo de ISQ identificados en pacientes con cierre esternal primario son también variables predictoras de ISQ en pacientes con CED.

\section{Pacientes y Métodos}

Estudio de casos y controles que incluyó a los pacientes bajo 15 años de edad, portadores de cardiopatía congénita y sometidos a cirugía cardíaca con CED, desde enero de 2009 a diciembre de 2010, en el Hospital Clínico de la Pontificia Universidad Católica de Chile (PUC), que es uno de los centros de referencia nacional para la resolución de las cardiopatías congénitas operables en pacientes bajo 15 años de edad.

Se definió como casos a aquellos pacientes con CED que presentaron ISQ y controles aquellos que no presen- taron infección dentro del mismo período. El diagnóstico de ISQ se realizó según los criterios diagnósticos vigentes del Centro para el Control y Prevención de Enfermedades (CDC) de Estados Unidos de América, publicadas el año 1999 y que fueron identificadas y registradas por el Comité de Prevención y Control de Infecciones Asociados a la Atención en Salud (IAAS) del hospital ${ }^{1}$.

Se excluyeron aquellos pacientes sometidos a cirugía cardíaca por patología infecciosa (endocarditis o mediastinitis), a los fallecidos antes del día 15 post operatorio $\mathrm{y}$ a aquellos con un seguimiento menor de 15 días post cirugía. Se realizó una revisión de las fichas clínicas, registrando variables demográficas (sexo, edad y peso al momento de la cirugía), tipo de cardiopatía congénita, puntaje de RACHS-1, (del inglés risk adjustment in congenital heart surgery), ampliamente utilizado en la estratificación de riesgo de las diferentes cirugías cardíacas de las cardiopatías congénitas (puntaje: 1-2 bajo riesgo, 3-4 mediano riesgo y 5-6 alto riesgo de mortalidad ${ }^{24}$, cirugía realizada y evolución peri-operatoria (días de hospitalización pre operatoria, infecciones previas, profilaxis antimicrobiana, días de hospitalización en Unidad de Cuidados Intensivos durante el post-operatorio, días de uso de fármacos vasoactivos, días de uso de VMI, uso de nutrición parenteral, indicación y tiempo de tórax abierto, procedimientos invasores previos, tiempo de CEC, necesidad de re-operación). Se registró además la presencia y tipo de ISQ, resultados de cultivos y tratamiento realizado.

El estudio fue aprobado por el Comité de Ética de la Dirección de Investigación y la Dirección del Hospital Clínico de la PUC, Red de Salud UC Christus.

\section{Análisis estadístico}

Las variables categóricas fueron descritas en términos de número y porcentaje, y las variables continuas en términos de mediana y rango. En el análisis univariable, la comparación entre variables categóricas se realizó mediante el test de $\chi^{2}$ o test exacto de Fisher, según correspondiera. En el análisis multivariable se utilizó un modelo de regresión logística, incluyendo sólo aquellas variables con 15 o más puntos porcentuales de diferencia entre casos y controles en el análisis univariado; sus resultados fueron expresados en términos de odds ratio ajustados y su respectivo intervalo de confianza $95 \%$. La bondad de ajuste del modelo se evaluó mediante el test de Hosmer y Lemeshow. Se consideró estadísticamente significativo todo valor-p $<0,05$. Los análisis fueron realizados mediante el software estadístico SPSS versión 22.

\section{Resultados}

En el período comprendido entre enero de 2009 y diciembre de 2010 se realizaron 514 cirugías cardíacas en nuestro hospital. En 67 pacientes se realizó cierre 
esternal diferido (13\%). De ellos, se excluyeron nueve pacientes: dos cuya indicación quirúrgica fue patología infecciosa (mediastinitis y endocarditis, respectivamente), cuatro fallecidos antes de los 15 días de post-operatorio ( $\sin$ antecedente de ISQ) y tres por registros incompletos. Los 58 pacientes restantes fueron incluidos en el análisis, $57 \%$ de sexo masculino.

La mediana de edad de la muestra fue de 9,5 días (2 días a 11 años). El 77\% de los pacientes tenían menos de un mes de edad y $19 \%$ pesaban $\leq$ tres kilos al momento de la cirugía.

Los diagnósticos más frecuentes fueron transposición de grandes arterias (TGA) $(21 / 58,36 \%)$ e hipoplasia de ventrículo izquierdo (HVI) (16/58, 27\%). Respecto al puntaje de RACHS-1, categorías de bajo riesgo 1 y 2 $(5 / 58,8,6 \%)$, categorías de mediano riesgo 3 y $4(31 / 58$, $53,4 \%)$ y categorías de alto riesgo 5 y $6(22 / 58,38 \%)$.

La mediana de tiempo de hospitalización pre cirugía fue de siete días (1 a 101 días) y tiempo de hospitalización en UCI post operatoria mediana de 10 días ( 5 a 90 días).

Diecinueve pacientes $(32 \%)$ recibieron antimicrobianos en dosis terapéutica previos a la cirugía, 10 de ellos por sospecha de infección connatal y nueve consideradas como IAAS.

La duración promedio del tiempo quirúrgico fue de 6,6 h, con una mediana de tiempo de circulación extracorpórea (CEC) de $207 \mathrm{~min}$. La principal indicación de cierre esternal diferido fue definido como profiláctica buscando los beneficios de esta técnica: evitar el efecto constrictivo torácico asociado al edema pulmonar y cardíaco post CEC y facilitar el manejo de los pacientes con alto riesgo de sangrado durante el post operatorio.

Todos los pacientes recibieron profilaxis antimicrobiana peri-operatoria consistente en cefazolina (o cefazolina + aminoglucósido en 55\% de los pacientes), la cual se mantuvo hasta $24 \mathrm{~h}$ post cierre esternal.

El tiempo promedio de cierre esternal diferido fue de tres días ( 1 a 23 días). Catorce pacientes (24\%) requirieron re-exploración torácica, en la mayoría de los casos por sangrado y taponamiento cardíaco (6 pacientes, 10,3\%). El cierre esternal definitivo, fue realizado en su mayoría en la UCIP (64\%) con el objetivo de evitar el potencial compromiso hemodinámico que significa el traslado de estos pacientes al pabellón quirúrgico.

La mediana de días de fármacos vasoactivos (DVA) y VMI post-cirugía fue de 6 días (Tabla 1).

Trece pacientes presentaron ISQ (22\%); 10 incisional superficial (17\%), uno incisional profunda $(1,7 \%)$ y dos mediastinitis (3,5\%). En cinco pacientes el diagnostico de ISQ fue clínico ya que no se aisló microorganismo en los cultivos. Las características clínicas y microbiológicas se detallan en la Tabla 2.

En el análisis univariado la única variable asociada significativamente con ISQ fue CEC mayor de $200 \mathrm{~min}$

\begin{tabular}{|c|c|}
\hline Variable & n (\%) o mediana (rango) \\
\hline \multicolumn{2}{|l|}{ Sexo } \\
\hline Varones & $33(56,9)$ \\
\hline Mujeres & $25(43,1)$ \\
\hline Edad & 9,5 días (2 días-11 años) \\
\hline \multicolumn{2}{|l|}{ Edad } \\
\hline Menor o igual a 7 días & $17(29,3)$ \\
\hline Mayor a 7 días & $41(70,7)$ \\
\hline Peso & $3.424 \mathrm{~g}(2.180 \mathrm{~g}-32.200 \mathrm{~g})$ \\
\hline \multicolumn{2}{|l|}{ Peso* } \\
\hline Menor o igual a $3.000 \mathrm{~g}$ & $10(18,9)$ \\
\hline Mayor a $3.000 \mathrm{~g}$ & $43(81,1)$ \\
\hline \multicolumn{2}{|l|}{ Diagnóstico } \\
\hline D-TGA & $21(36,2)$ \\
\hline $\mathrm{HVI}$ & $16(27,6)$ \\
\hline Otro & $21(36,2)$ \\
\hline \multicolumn{2}{|l|}{ RACHS-1 } \\
\hline $1-4$ & $36(62,1)$ \\
\hline $5-6$ & $22(37,9)$ \\
\hline \multicolumn{2}{|l|}{ Hospitalización pre-cirugía } \\
\hline Menor o igual a 7 días & $31(53,4)$ \\
\hline Mayor a 7 días & $27(46,6)$ \\
\hline \multicolumn{2}{|l|}{ Procedimiento pre-quirúrgico } \\
\hline Sí & $18(31,0)$ \\
\hline No & $40(69,0)$ \\
\hline \multicolumn{2}{|l|}{ CEC } \\
\hline Menor o igual a $200 \mathrm{~min}$ & $27(46,6)$ \\
\hline Mayor a $200 \mathrm{~min}$ & $31(53,4)$ \\
\hline \multicolumn{2}{|l|}{ Tórax abierto } \\
\hline Menor o igual a 2 días & $28(48,3)$ \\
\hline Mayor a 2 días & $30(51,7)$ \\
\hline \multicolumn{2}{|l|}{$\mathrm{VMI}$} \\
\hline Menor o igual a 5 días & $24(41,4)$ \\
\hline Mayor a 5 días & $34(58,6)$ \\
\hline \multicolumn{2}{|l|}{ Re-exploración post-quirúrgica } \\
\hline Sí & $14(24,1)$ \\
\hline No & $44(75,9)$ \\
\hline \multicolumn{2}{|l|}{ Infección del sitio quirúrgico } \\
\hline Sí & $13(22,4)$ \\
\hline No & $45(77,6)$ \\
\hline
\end{tabular}

$(\mathrm{p}=0,013)$ (Tabla 3). Las variables que obtuvieron 15 o más puntos porcentuales de diferencia entre el grupo de casos y controles fueron CEC, VMI, necesidad de re-exploración quirúrgica, puntaje de RACHS-1 y diagnóstico. Estas se incluyeron en un análisis multivariado por regresión logística, en el que CEC mayor a $200 \mathrm{~min}$ 


\begin{tabular}{|c|c|c|}
\hline Paciente & Resultado cultivo & $\begin{array}{l}\text { Tipo de infección } \\
\text { del sitio quirúrgico }\end{array}$ \\
\hline 1 & $\begin{array}{l}\text { Herida operatoria y secreción traqueal: } \\
\text { Pseudomonas aeruginosa y Klebsiella } \\
\text { pneumoniae } \mathrm{BLEE}(+)\end{array}$ & Mediastinitis \\
\hline 2 & $\begin{array}{l}\text { Herida operatoria: Staphylococcus coagulasa } \\
\text { negativa oxacilina resistente }\end{array}$ & Incisional superficial \\
\hline 3 & $\begin{array}{l}\text { Herida operatoria: Staphylococcus coagulasa } \\
\text { negativa oxacilina resistente }\end{array}$ & Incisional superficial \\
\hline 4 & $\begin{array}{l}\text { Herida operatoria: Staphylococcus coagulasa } \\
\text { negativa oxacilina resistente }\end{array}$ & Incisional superficial \\
\hline 5 & $\begin{array}{l}\text { Herida operatoria: Staphylococcus coagulasa } \\
\text { negativa oxacilina resistente }\end{array}$ & Incisional superficial \\
\hline 6 & Negativo & Incisional superficial \\
\hline 7 & Negativo & Incisional superficial \\
\hline 8 & Negativo & Incisional profundo \\
\hline 9 & Negativo & Incisional superficial \\
\hline 10 & $\begin{array}{l}\text { Herida operatoria: Staphylococcus aureus } \\
\text { SM+ Staphylococcus coagulasa negativa } \\
\text { oxacilina resistente }\end{array}$ & Incisional superficial \\
\hline 11 & Negativo & Incisional superficial \\
\hline 12 & $\begin{array}{l}\text { Herida operatoria y líquido pleural: Candida } \\
\text { albicans }\end{array}$ & Mediastinitis \\
\hline 13 & $\begin{array}{l}\text { Herida operatoria: Staphylococcus coagulasa } \\
\text { negativa oxacilina resistente }\end{array}$ & Incisional superficial \\
\hline
\end{tabular}

(OR ajustado=9,53; IC 95\% 1,37-66,35) y VMI más de cinco días (OR ajustado $=8,98$; IC 95\% 1,16-69,40) se asociaron de manera significativa e independiente a ISQ. El modelo de regresión logística ajustó adecuadamente los datos (valor-p test de Hosmer y Lemeshow $=0,971$ ).

\section{Discusión}

El presente estudio permitió establecer la tasa de ISQ que se presentó en pacientes portadores de cardiopatía congénita sometidos a cirugía cardíaca con CED en uno de los centros pediátricos de cirugía cardíaca perteneciente a la red de Garantías Explícitas de Salud (GES) del Ministerio de Salud, actualmente a cargo de la atención de, al menos, $20 \%$ de los niños chilenos con cardiopatías congénitas.

Las tasas de ISQ en cirugía cardíaca pediátrica reportadas por el Comité de Prevención y Control de IAAS local durante los años 2009 y 2010 fueron de 4,8 y 4,9\%, respectivamente (Tasa MINSAL 2007 P50
Tabla 3. Comparación de variables demográficas y perioperatorias entre casos y controles (análisis univariable)

\begin{tabular}{|c|c|c|c|}
\hline \multirow[b]{2}{*}{ Variables } & \multicolumn{2}{|c|}{ Infección del sitio quirúrgico } & \multirow[b]{2}{*}{ Valor $p$} \\
\hline & $\begin{array}{c}\text { Presente } \\
\text { (n: 13) } \\
\text { n (\%) }\end{array}$ & $\begin{array}{c}\text { Ausente } \\
(\mathrm{n}: 45) \\
n(\%)\end{array}$ & \\
\hline \multicolumn{4}{|l|}{ Sexo } \\
\hline Varones & $6(46,2)$ & $27(60,0)$ & 0,526 \\
\hline Mujeres & $7(53,8)$ & $18(40,0)$ & \\
\hline
\end{tabular}

Edad

$\begin{array}{lccc}\text { Menor o igual a } 7 \text { días } & 3(23,1) & 14(31,1) & 0,736 \\ \text { Mayor a } 7 \text { días } & 10(76,9) & 31(68,9) & \end{array}$

Peso*

Menor o igual a $3.000 \mathrm{~g} . \quad 2(16,7) \quad 8(19,5) \quad 1,000$

Mayor a $3.000 \mathrm{~g} . \quad 10(83,3) \quad 33(80,5)$

$\begin{array}{llll}\text { Diagnóstico } & & & \\ \text { D-TGA } & 5(38,5) & 16(35,6) & 0,463 \\ \text { HVI } & 5(38,5) & 11(24,4) & \\ \text { Otras } & 3(23,1) & 18(40,0) & \end{array}$

RACHS-1

$\begin{array}{llll}1-4 & 6(46,2) & 30(66,7) & 0,208 \\ 5-6 & 7(53,8) & 15(33,3) & \end{array}$

Hospitalización pre-cirugía

$\begin{array}{llll}\text { Menor o igual a } 7 \text { días } & 7(53,8) & 24(53,3) & 1,000\end{array}$

Mayor a 7 días $\quad 6(46,2) \quad 21(46,7)$

Procedimiento pre-quirúrgico

$\begin{array}{lccc}\text { Sí } & 3(23,1) & 15(33,3) & 0,735 \\ \text { No } & 10(76,9) & 30(66,7) & \end{array}$

$\begin{array}{lccc}\text { CEC } & & & \\ \text { Menor o igual a } 200 \mathrm{~min} & 2(15,4) & 25(55,6) & 0,013 \\ \text { Mayor a } 200 \mathrm{~min} & 11(84,6) & 20(44,4) & \end{array}$

Tórax abierto

$\begin{array}{llll}\text { Menor o igual a } 2 \text { días } & 5(38,5) & 23(51,1) & 0,534 \\ \text { Mayor a } 2 \text { días } & 8(61,5) & 22(48,9) & \end{array}$

VMI

$\begin{array}{lccc}\text { Menor o igual a } 5 \text { días } & 2(15,4) & 22(48,9) & 0,053\end{array}$

Mayor a 5 días $\quad 11(84,6) \quad 23(51,1)$

Re-exploración post-quirúrgica

$\begin{array}{lccc}\text { Sí } & 5(38,5) & 9(20,0) & 0,268 \\ \text { No } & 8(61,5) & 36(80,0) & \end{array}$

* 5 datos faltantes. D-TGA: d-transposición de grandes arterias, HVl: hipoplasia del ventrículo izquierdo, CEC: circulación extracorpórea, VMI: ventilación mecánica invasora. 
de 3,4\%). La tasa observada de ISQ en nuestros pacientes con CED fue $22 \%$, mayor a lo reportado en la literatura médica, lo que confirma a estos pacientes como un grupo de alto riesgo. Estudios publicados desde los años 80 reportan cifras de hasta $28 \%{ }^{21}$. Sin embargo, la incidencia reportada en estudios posteriores es menor (15,2\%). Las series más numerosas publicadas recientemente por Tabutt y cols. ${ }^{21}$ y Harder y cols. ${ }^{22}$, reportan incidencias de ISQ de 6,7 y $11 \%$, respectivamente.

En relación al diagnóstico de ISQ, en cinco pacientes (38\%) se planteó este diagnóstico considerando sólo las características clínicas de la herida operatoria, ya que sus cultivos fueron negativos.

En nuestro Centro se utilizó CED en $100 \%$ de los pacientes portadores de hipoplasia del ventrículo izquierdo sometidos a la primera etapa de la cirugía paliativa (Norwood Sano) y en más de $75 \%$ de aquellos portadores de transposición de grandes arterias, a los que se les realizó switch arterial. Este hecho podría relacionarse con la tasa de ISQ en nuestros pacientes con CED según los datos obtenidos por el estudio multicéntrico de Johnson y cols., quienes reportaron que centros con uso medio o alto de CED en relación a la etapa 1 de la cirugía paliativa de la HVI (más de 75\% de los pacientes) tienen significativamente mayor incidencia de ISQ ${ }^{18}$.

La incidencia de mediastinitis en nuestros pacientes alcanzó a 3,5\%, frecuencia similar a la descrita en estudios más recientes, los que reportan tasas de 1,9 a $7,5 \%$, $8,15,21-23,25$.

El análisis de regresión logística demostró que el tiempo de CEC mayor a 200 min y el tiempo en VMI por más de 5 días fueron los principales factores de riesgo asociado con la ocurrencia de ISQ con un OR ajustado = 9,53 (IC 95\% 1,37-66,35) y OR ajustado = 8,98 (IC 95\% 1,16-69,40), respectivamente. La mediana de días de VMI y de tiempo de CEC en nuestros pacientes es similar a lo reportado en la literatura científica ${ }^{18}$.

Otros autores no han encontrado esta asociación ${ }^{21}$. El diseño de este estudio no permite atribuir causalidad a la asociación encontrada.

El reducido número de pacientes incluidos y el diseño retrospectivo constituyen nuestra mayor limitación en el presente análisis.

Es ampliamente reconocido que la respuesta inflamatoria sistémica observada en niños sometidos a cirugía cardíaca y CEC está asociada a alto riesgo de falla orgánica múltiple ${ }^{26,27}$. La etiología es multifactorial e incluye el trauma quirúrgico, injuria por isquemia reperfusión y activación leucocitaria producida por el circuito. Está demostrado que la ultrafiltración disminuye este fenómeno pero no lo anula. Al parecer, en los pacientes con hipoplasia del ventrículo izquierdo, la respuesta inflamatoria sería mayor en comparación con otras cardiopatías ${ }^{26,27}$. Las concentraciones plasmáticas de citokinas pro-infla- matorias se han asociado directamente a la duración de la CEC y a ventilación mecánica prolongada ${ }^{26,27}$. Por otra parte, la apoptosis e inhibición linfocitaria, probablemente secundaria al estado hiperinflamatorio post-CEC, podría determinar un estado de inmunodeficiencia transitoria que aumentaría el riesgo de infección ${ }^{28}$, lo que podría sustentar la asociación encontrada.

El énfasis en este grupo de riesgo, se debe centrar en el desarrollo de protocolos claramente orientados a la prevención de infecciones del sitio quirúrgico ${ }^{29}$.

\section{Conclusión}

La tasa de ISQ en los pacientes portadores de cardiopatía congénita sometidos a cirugía cardíaca con CED fue de $22 \%$, constituyendo un grupo de riesgo. Los factores de riesgo identificados asociados a la ocurrencia de ISQ fueron CEC mayor a 200 min y el tiempo de VMI mayor de 5 días.

Estos hallazgos son un importante aporte para el desarrollo de protocolos estandarizados en el cuidado de niños sometidos a cirugía cardíaca con CED, con el claro objetivo de disminuir esta tasa, y con especial énfasis en una conducta activa en el cierre esternal y retiro precoz de la VMI.

\section{Resumen}

Introducción: Las infecciones del sitio quirúrgico (ISQ) son importante causa de morbilidad en cirugía cardíaca pediátrica. Los factores de riesgo en pacientes que requieren cierre esternal diferido (CED) se desconocen. Objetivos: Reportar la tasa de ISQ en niños sometidos a cirugía cardíaca con CED y determinar factores de riesgo de ISQ. Metodología: Estudio retrospectivo de casos y controles en pacientes bajo 15 años de edad, sometidos a cirugía cardíaca con CED, en los años 2009 y 2010. Se consideró casos aquellos con ISQ diagnosticada según criterios del comité de IAAS local. Se realizó análisis uni y multivariado de las variables. Se consideró significativo un $\mathrm{p}<0,05$. Resultados: Se incluyeron 58 pacientes; la mediana de edad fue 9,5 días. Diagnósticos más frecuentes fueron transposición de grandes arterias $(36 \%)$ e hipoplasia de ventrículo izquierdo $(27 \%)$. Trece pacientes presentaron ISQ (22\%); 11 incisionales y 2 mediastinitis. Se asociaron de manera independiente a ISQ: circulación extracorpórea (CEC) mayor a $200 \mathrm{~min}$ (OR ajustado $=9,53$; IC 95\% 1,37-66,35) y ventilación mecánica invasora (VMI) más de 5 días (OR ajustado $=8,98$; IC 95\% 1,16-69,40). Conclusión: La duración de CEC y VMI son factores de riesgo de ISQ en niños sometidos a cirugía cardíaca con CED. 


\section{Referencias bibliográficas}

1.- Mangram A J, Horan T C, Pearson M L, Silver L C, Jarvis W R. The Hospital Infection Control Practices Advisory Committee. Guideline for the prevention of surgical site infection, 1999. Infect Control Hosp Epidemiol 1999; 20: 247-80.

2.- Barriga J, Cerda J, Abarca K, Ferrés M, Fajuri P, Riquelme M, et al. Infecciones asociadas a la atención en salud (IAAS) en pacientes pediátricos post-operados de cardiopatías congénitas. Rev Chilena Infectol 2014; 31 (1): 16-20.

3.- Klevens R M, Edwards J R, Richards C L Jr, Horan T C, Gaynes R P, Pollock D A, et al. Estimating health care-associated infections and deaths in US hospitals, 2002. Public Health Rep 2007; 122: 160-6.

4.- Welke K F, Shen I, Ungerleider R M. Current assessment of mortality rates in congenital cardiac surgery. Ann Thorac Surg 2006; 82 (1): 164-70.

5.- Grisaru-Soen G, Paret G, Yahav D, Boyko V, Lerner-Geva L. Nosocomial infections in pediatric cardiovascular surgery patients: a 4-year survey. Pediatr Crit Care Med 2009; 10 (2): 202-6.

6.- Pollock E M, Ford-Jones E L, Rebeyka I, Mindorff C M, Bohn D J, Edmonds J F, et al. Early nosocomial infections in pediatric cardiovascular surgery patients. Crit Care Med 1990; 18 (4): 378-84.

7.- Furnary A P, Magovern J A, Simpson K A, Magovern G J. Prolonged open sternotomy and delayed sternal closure after cardiac operations. Ann Thorac Surg 1992; 54: 233-9.

8.- Woodward C S, Son M, Calhoon J, Michalek J, Husain S A. Sternal wound infections in pediatric congenital cardiac surgery: a survey of incidence and preventative practice. Ann Thorac Surg 2011; 91 (3): 799-804.

9.- Allpress A L, Rosenthal G L, Goodrich K M, Lupinetti F M, Zerr D M. Risk factors for surgical site infections after pediatric cardiovascular surgery. Pediatr Infect Dis J 2004; 23: 231-4.

10.- Mehta P A, Cunningham C K, Colella C B, Alferis G, Weiner L B. Risk factors for sterna wound and other infections in pediatric cardiac surgery patients. Pediatr Infect Dis J 2000; 19 : 1000-4.

11.- Kagen J, Lautenbach E, Bilker W B, Matro J, Bell L M, Domínguez T E, et al. Risk factors for mediastinitis following median sternotomy in children. Pediatr Infect Dis J 2007; 26: 613-8.

12.- Nateghian A, Taylor G, Robinson J L. Risk factors for surgical site infections following open-heart surgery in a Canadian pediatric population. Am J Infect Control 2004; 32: 397-401.

13.- Costello J M, Graham D A, Morrow D F, Morrow J, Potter-Bynoe G, Sandora T J, et al. Risk factors for surgical site infection after cardiac surgery in children. Ann Thorac Surg 2010; 89 (6): 1833-41

14.- Sohn A H, Schwartz J M, Yang K Y, Jarvis W R, Guglielmo B J, Weintrub P S. Risk factors and risk adjustment for surgical site infections in pediatric cardiothoracic surgery patients. Am J Infect Control 2010; 38 (9): 706-10.

15.- Riphagen $\mathrm{S}, \mathrm{McD}$ ougall M, Tibby $\mathrm{S} M$, Alphonso N, Anderson D, Conal A, et al. "Early" delayed sterna closure following pediatric cardiac surgery. Ann Thorac Surg 2005; 80: 678-85

16.- Srinivasan C, Sachdeva R, Morrow W R, Gossett J, Chipman C W, Imamura M, et al. Standardized management improves outcomes after the Norwood procedure. Congenit Heart Dis 2009; 4 (5): 329-37.

17.- Das S, Rubio A, Simsic J M, Kirshbom P M, Kogon B, Kanter K R, at al. Bloodstream infections increased after delayed sternal closure: cause or coincidence. Ann Thorac Surg 2011; 91 (3): 793-7.

18.- Johnson J N, Jaggers J, Li S, O’Brien S M, Li J S, Jacobs J P, et al. Center variation and outcomes associated with delayed sternal closure after stage 1 palliation for hypoplastic left heart syndrome. J Thorac Cardiovasc Surg 2010; 139 (5): 1205-10.

19.- Sarris G E, Chatzis A C, Giannopoulos N M, Kirvassilis G, Berggren H, Hazekamp M, et al. The arterial switch operation in Europe for transposition of the great arteries: a multi-institutional study from the European Congenital Heart Surgeons Association. J Thorac Cardiovasc Surg 2006; 132 (3): 633-9.
20.- Erek E, Yalcinbas Y, Turkekul Y, Saygili A Ayse Ulukol, Ayse Sarioglu, et al. Indications and risks of delayed sternal closure after open heart surgery in neonates and early infants. World J Pediatr Congenit Heart Surg 2012; 3 (2): 229-35.

21.- Tabbutt S, Duncan B W, McLaughlin D, Wessel D L, Jonas R A, Laussen P C. Delayed sternal closure after cardiac operations in a pediatric population. J Thorac Cardiovasc Surg 1997; 113 (5): 886-93.

22.- Harder E E, Gaies M G, Yu S, Donohue J E, Hanauer D A, Goldberg C S, et al. Risk factors for surgical site infection in pediatric cardiac surgery patients undergoing delayed sternal closure. J Thorac Cardiovasc Surg 2013; 146 (2): 326-33.

23.- McElhinney D B, Reddy V M, Parry A J, Johnson L, Fineman J R, Hanley F L. Management and outcomes of delayed sternal closure after cardiac surgery in neonates and infants. Crit Care Med 2000; 2 8(4): 1180-4.

24.- Jenkins K J, Gauvreau K, Newburger J W, Spray T, Moller J H, Iezzoni L. Consensusbased method for risk adjustment for surgery for congenital heart disease. J Thorac Cardiovasc Surg 2002; 123: 110-6.

25.- Al-Sehly A A, Robinson J L, Lee B E, Taylor G, Ross D B, Robertson M, et al. Pediatric post sternotomy mediastinitis. Ann Thorac Surg 2005; 80 (6): 2314-20.

26.- Appachi E, Mossad E, Mee R B, Bokesch P. Perioperative serum interleukins in neonates with hypoplastic left-heart syndrome and transposition of the great arteries. J Cardiothorac Vasc Anesth 2007; 21 (2): 184-90.

27.- Allan C K, Newburger J W, McGrath E, Elder J, Psoinos C, Laussen P C, et al. The relationship between inflammatory activation and clinical outcome after infant cardiopulmonary bypass. Anesth Analg 2010; 111 (5): 1244-51

28.- Shi S S, Shi C C, Zhao Z Y, Shen H Q, Fang X M, Tan L H, et al. Effect of open heart surgery with cardiopulmonary bypass on peripheral blood lymphocyte apoptosis in children. Pediatr Cardiol 2009; 30 (2): 153-9.

29.- Woodward C, Son M, Taylor R. Prevention of sternal wound infection in pediatric cardiac surgery: A protocolized approach. World J Pediatr Congenit Heart Surg 2012; 3 (4): 463-9. 